\title{
MOBILITY MODELS FOR DELAy TOLERANT NETWORK: A SURVEY
}

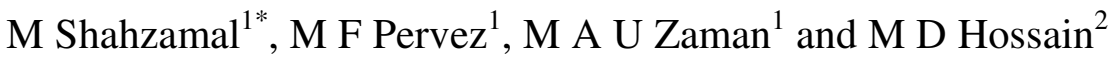 \\ ${ }^{1}$ Institute of Electronics, Bangladesh Atomic Energy Commission, Bangladesh \\ ${ }^{2}$ Institute of Computer Science, Bangladesh Atomic Energy Commission, Bangladesh
}

\begin{abstract}
Delay Tolerant Network (DTN) is an emerging networking technology that is widely used in the environment where end-to-end paths do not exist. DTN follows store-carry-forward mechanism to route data. This mechanism exploits the mobility of nodes and hence the performances of DTN routing and application protocols are highly dependent on the underlying mobility of nodes and its characteristics. Therefore, suitable mobility models are required to be incorporated in the simulation tools to evaluate DTN protocols across many scenarios. In DTN mobility modelling literature, a number of mobility models have been developed based on synthetic theory and real world mobility traces. Furthermore, many researchers have developed specific application oriented mobility models. All these models do not provide accurate evaluation in the all scenarios. Therefore, model selection is an important issue in DTN protocol simulation. In this study, we have summarized various widely used mobility models and made a comparison of their performances. Finally, we have concluded with future research directions in mobility modelling for DTN simulation.
\end{abstract}

\section{KEYWORDS}

Delay Tolerant Networking, Mobility Modelling, DTN Simulation,

\section{INTRODUCTION}

Delay tolerant network (DTN) is increasingly becoming popular due to its capability to be deployed with low cost in the environments where end-to-end connectivity is not normal. In DTNs, the messages are delivered from source to destination via intermediate mobile nodes that store, carry and forward data as the contact opportunity arises. Therefore, the underlying mobility patterns of the nodes in such a network will determine the performance of the network. However, it is difficult to evaluate DTN protocols and applications in real world environment. Thus, the performances of DTN protocols are usually evaluated using simulator based on different mobility models. These mobility models are expected to reflect the real mobility patterns of nodes [1]-[2].

In DTN applications, message transmission occurs only when appropriate relays meet each other. The time elapsed between such two meetings is the basic metric for characterizing mobility in DTNs. The time taken by a node to first meet other node is defined as the meeting time. Once two nodes come into communication range of each other, they start processes to exchange data. The time duration they will exchange data depends on the time they remain in the communication range of each other. This time duration is defined as the contact time. If they do not finish exchanging the packet during the contact time (due to contention in the network), then they require waiting till they come into communication range again. This time duration is termed as the inter-meeting time. Therefore, a realistic performance analysis of any mobility assisted routing scheme requires a knowledge of statistics of these three quantities. There have been a 
number of studies regarding these microscopic mobility properties and its characteristics. As a result, today's mobility models are perfectly capable to assume these properties [3].

Mobility models are developed using practical traces and some synthetic theories that try to achieve realism. Many traditional mobility models are still widely used to support easy DTN protocol evaluation. They usually cover only selected mobility characteristics whereas synthetically generated node mobility models allow for fine-tuning in many respects. We have grouped simplest traditional models as Random Mobility Models. In the literature, there are a number of such mobility models. Among them, the following popular models will be discussed in this paper.

1. Random Walk (RW) Model: A simple mobility model that relay on random speeds in random directions.

2. Random Walkway Point (RWP) Model: A model that add pause times when nodes assume new destination and speed.

3. Random Direction (RD) Mobility Model: A model that drive nodes up to the boundary of the simulation area before changing direction and speed.

4. Levy Walks (LW) Mobility Model: A model that derives travel lengths and pause times from a power law distribution.

Applications of the synthetic models on the map derived another mobility models class where random pattern is constrained within the map. In these models, map data is included using a subset of the Well Known Text (WKT) format. In fact, WKT is based on ASCII format that is frequently used in Geographic Information System (GIS) programs [4]. We have grouped these models as Map-Constrained Mobility Models. The following are some of the popular map-based mobility models.

1. Map-Based Mobility Models (MBM): A model that moves nodes with random speed and directions following a map.

2. Shortest Path-Based Map Mobility Model (SPBMM): A model that is similar to MBM, but selects a certain destination in the map for all nodes and finds the shortest path to reach the destination.

3. Route-Based Map Mobility Model (RBM): A model that have predetermined routes on the map that they travel.

4. Manhattan Mobility Model (MMM): A model that formulates a grid like structure of horizontal and vertical lines on map to drive nodes with predefined probability.

In many DTN applications, wireless nodes are carried by humans, animals, vehicles, or planets which possess their own movement patterns. DTN routing protocols often exploit these patterns to implement applications [5]. In the human community, people meet strangers by chance or familiar strangers due to having similar mobility patterns while family, friends and colleagues by intention [3]. Therefore, human mobility pattern also has characteristic like macroscopic structure along with microscopic mobility properties. Thus, many researchers have developed mobility models based on human behaviour. We have grouped these models as Social Mobility Models. We have analysed the following popular models.

1. Community-Based Mobility Model (CMM): A model that is directly driven by a social network.

2. Time-Variant Community Model (TVCM): A model that derives nodes from one community to another with a fixed global velocity and use transition probabilities.

3. Working Day Movement (WDM) Model: A model that accounts all activities done by human during a working week. 
4. General Social Mobility Model (GeSoMo): A model that separate the core mobility models from the structural explanation of the social network.

Since mobility models can be configured on per node basis, it is possible to combine multiple mobility models in one simulation. Consequently, researchers have motivated to create mobility models where, for example, in a shopping centre or a park some nodes move following map along roads and others who walk around randomly within. We have included such mobility models into Composite Mobility Models group. The following are the very familiar and successful mobility models of this type.

1. Self-similar Least-Action Walk (SLAW) Model: A model that accounts all the five statistical features as well as user-created virtual ones.

2. Post-Disaster Mobility (PDM) Model: A mode that mimics real scenario and focus on role-based movements based on map.

3. Localized Random Walk (LRAW) Mobility Model: A model that places nodes to cells and drive nodes counting distance from those cells.

4. Contact Graph (CG) Based Mobility Model: A model that involves tools and metrics from social network analysis and graph theory.

In our study, we have used different notions and terminology to represent the properties of DTN. That is described in this paragraph. A node is here denoted as a wireless enabled device integrating at least one interface. Node position describes the specific location of a node in relation to a grid. The mobility features that capture a node movement in mobility models are a node's speed and also a direction. The pause time of a node relates to the time period that a node is steady in a specific position, i.e., the interval of time when the node's speed is zero or close to zero. Inter-contact time indicates the time duration between two successive meetings of the same two nodes. As for contact duration, this refers to the time period two nodes attain while within the same radio range.

The purpose of this study is to provide an overview about the available mobility models used in simulator and a comparison of their performance as it affects the performance of DTN routing protocols significantly. We have presented different characteristics of each model. These characteristics are frequently applicable when one selects mobility models to evaluate their designed DTN protocols. In addition, we have focused to find out what are the potential future research issues to develop more realistic mobility models. We have organized the papers as follows. In the section 2 , we have presented descriptions of each mobility model characteristics. The comparisons of performance of model have been described in the section 3. Future research directions have been provided in the section 4 . Finally, we have concluded our observation in section 5 .

\section{DTN MOBILITY MODELS}

\subsection{Random Mobility Models}

\subsubsection{Random Walk (RW) Model}

Random walk mobility model is based on the argument that entities naturally move around in unpredictable ways. In this model, every node moves towards a new randomly chosen location. A random direction and speed is assigned to each node from a predefined range and nodes of a network are independent from one another [3]. A new direction is again assigned from predefined ranges whenever any node reaches the destination location. In this model, the distributions of mobility parameters are a function of time. As the mobility parameters achieve the stable state of 
distributions, the simulation produces consistent results. This has kept its popularity to evaluate DTN protocols. However, the variations of direction and speed in this model are limited in a certain ranges that are defined beforehand. Besides, the RW Models produce memory-less mobility pattern because it cannot keep records of previous patterns formed by the locations and speed values of mobile nodes [6]. The current speed and direction of a node is independent of its past speed and direction as well. These characteristics can generate unrealistic movements such as sudden stops and sharp turns. A variant of this model, namely Gauss-Markov Mobility Model, has been introduced to resolve this discrepancy [7].

\subsubsection{Random Walkway Point (RWP)}

Another basic and simplest model that is frequently used in simulation is the Random Walkway Point (RWP). RWP includes a random pause time after finishing each movement segments in the random walk. In RWP, mobility model direction angles, speeds and pause times are sampled from a uniform distribution. In simulation, random coordinates over simulation area are assigned to the nodes. Nodes move directly to the specified destination at constant speed, stay there few moments, and then acquire a new, random, destination. This continues throughout the simulation and nodes run along these zig-zag paths based on RWP model. This model is mathematically analysable and easy to implement. Thus, the researchers can easily test their own algorithms using it. This creates simple, but quite artificial movement which humans hardly can move or select for their destinations in a square area. This model often results in a non-uniform stationary node distribution and hence complicates the analysis [8, 9, 10, 11]. RWP is improved by Smooth Random Mobility Model (SRMM) that adds a temporal dependency where speed is changed incrementally in a smooth fashion [12].

\subsubsection{Random Direction (RD) Mobility Model}

The random direction distribution overcomes the problem of non-uniform distribution found in RWP [13]. In the RD model, a mobile node gets a movement degree randomly and moves in a particular direction until it touches the destination boundary of simulation area with a specified speed. When it travels to boundary area, it stops for a specified pause time before choosing a new direction to move again. This model is more consistent in comparison with other random models and offers quantitatively even distribution of nodes in the simulation area. Many variants of the RD mobility model have been developed. The first variant proposed by Royer et al. [14] assign a travel duration to each node before it starts to move with a random speed in a random direction. It then stops and changes its direction if the assigned travel duration expires before it reaches the border of the simulation area. In such a case, a new direction is uniformly chosen in $[0,2 \pi]$. The second variant of the RD mobility model was presented by Haas [15]. In his work, when a node reaches a boundary of the simulation area, it instantaneously re-enter into the simulation area from the opposite boundary. It then continues to move with the previous speed and direction. The third variant presented by Bettstetter added that node would bounce off and continue to move with the previous speed in a new direction when a node reaches a boundary of the simulation area [13].

\subsubsection{Levy Walks (LW) Mobility Model}

This is very similar to random walk, except that the movement lengths and pause times are drawn from a power law distribution. This model is capable to produce almost same inter-contact time distributions as many real world traces. However, the model does not capture characteristics such as heterogeneity among nodes, repetitiveness, group mobility or any relationships between nodes. Another important characteristic of Levy walks is its high diffusivity. This means that the variance of the displacement between the current position at time $t$ and a previous position at time $\mathrm{t}_{0}$ is high [16]. The variant of LWM model is the Truncated Levy Walks (TLW). In TLW model, 
truncated Pareto distributions [17] is used for movement and pause-time distributions are used to emulate mobility within a confined area. In comparison to existing random mobility models, TLW can deliver more realistic representations of statistical patterns found in human mobility as well as it retains its simplicity and analytical tractability of random mobility models [18]. The author [18] observed that traces produced by volunteers moving in outdoor settings are quite similar with the patterns delivered by a model in which the movement lengths followed a truncated Levy distribution.

\subsection{Map-Constrained Mobility Models}

\subsubsection{Map-Based Mobility Models (MBM)}

The simple random Map-Based Mobility model (MBM) is a derivative of the Random Walk model. In this model, nodes move to randomly determined directions on the map following the roads as defined by the map data. In addition, it has options to select different node groups that use only certain parts of the map. By this way, it can distinguish between cars and pedestrians so that the former do not drive on pedestrian paths or inside buildings. When a simulation is done, nodes are randomly placed in the map area. These nodes move onward on the path segments until they touch the end of the road and turn back or end up in an intersection. In intersections, nodes that follow MBM select randomly a new direction but do not return back where they came from. When a node has travelled a configurable distance, it stops for a while (configurable), and then continues its journey [19]. The limitation of MBM is that nodes always move following the paths defined by the map data. This result in movement patterns of the nodes controlled by the map data and thus may not be a very accurate approximation of real human mobility [20].

\subsubsection{Shortest Path-Based Map Mobility Model (SPBMM)}

The improved version of MBM is the Shortest Path-Based Map Mobility Model. This model also initially places the nodes in random places of map area. However, all nodes travel to a certain destination in the map and follow Dijkstra's shortest path algorithm [21] to discover the shortest path to the destination. When nodes have reached the destination, they have to wait for a while and select a new destination. All the places in the map usually have same probability to be chosen as the next destination, but the map data can also contain Points of Interest (POIs). These POIs are divided into several POI groups. Every node group can have configurable probability for choosing POI in certain group as the next destination. POIs have advantages for modelling places where people tend to gather e.g. restaurants, supermarkets and tourist attractions [4]. SPBMM is found easy to understand and competent to use in simulations, but it does not assure inter-contact time and contact time distributions that match real world traces when small number of nodes is used in the simulation [22].

\subsubsection{Route-Based Map Mobility Model (RBMM)}

In this model, some nodes are assigned predetermined routes that they must travel on the map. This kind of Route-Based Models, RBMs, shows better performance in simulating nodes movement, especially, on the bus and tram routes. In this model, routes within the map contain many points and these points are termed as stops on the routes. Nodes wait on every stop for some time before traveling to the next stop. Nodes follow the shortest path approach to reach the destination [4]. Mobility model receives map data after converting through GIS programs and define both POIs and routes based on the map information. STreet Random Waypoint (STRAW) mobility Model is a variant of RBMM where nodes travel according to a realistic vehicular traffic model on roads defined by real street map data. This model also includes some essential and optional techniques for modelling vehicular motion [23]. 
International Journal of Wireless \& Mobile Networks (IJWMN) Vol. 6, No. 4, August 2014

\subsubsection{Manhattan Mobility Model (MMM)}

MMM is another map based mobility model that is widely used to simulate the movement pattern of mobile nodes on streets defined by maps. This model can be applied to imitate movement in an urban area where portable devices are supported with stabilized computing service. The map of MMM formulates a grid like structure of horizontal and vertical lines. This structure represents streets on the map. The mobile nodes are allowed to move along the grid in horizontal or vertical direction. The mobile node is allowed to change its direction at a predefined probability i.e. on the same street is 0.5 , turning left is 0.25 , turning right is 0.25 . Due to this probability pattern, the MMM might have high spatial dependence and high temporal dependence. This model applies too geographically restrictions on node mobility. However, it gives a node some freedom for changing its direction [24].

\subsection{Social Mobility Models}

\subsubsection{Community-based Mobility Model (CMM)}

The CMM is the first and flexible model which is directly drawn from a social network. In CMM, nodes are grouped as friends who belong to the same community and non-friends who are with different community. At the beginning, the movement area is divided into some regions as a grid and each community is assigned into a cell of the grid. A link is established between all the friend and non-friend nodes in the network which will be used later to drive node movements. In this model, nodes move between the communities based on node attraction feature [25]. The drawback of this model is gregarious behaviour of nodes. In this case, when a node has decided to exit the community, all other nodes of the community follow this node. The variant that resolve this issue by considering both node and location attraction is home-cell community-based mobility model (HCMM). HCMM introduces the idea of foreign community to mention that some nodes have also social links with communities other than the home. In this model, each node is initially is assigned to a specific community as well as each node has social links with all the other members of its home community. Some special nodes also have social links with foreign communities other than the home community [26]. The probability of moving a node from its home community towards a given community is proportional to the number of ties with nodes of the destination community [27].

\subsubsection{Time-variant Community Model (TVCM)}

In this model, the terrains (simulation plane) are divided into many sub terrains and each of which is designated as a community. At any instant, a specified node can be observed in any one of the communities. Nodes are assigned a fixed global velocity and travel from one community to another using transition probabilities, which follows a Markov Chain [28]. This whole arrangement of communities and their associated transition probabilities remains fixed over a time period of some duration. The node executes consequently different time periods until its original starting time period comes back. In this model, separate model can be assigned to each node or every node assumes the model like vanilla which they can build. This model captures skewed location preferences and that is considered as the essential components. In this case, an open terrain is modelled by considering that if an epoch falls out of the boundary of the terrain it has been absorbed. On the other hand, when it merely falls outside the boundary of the current community, it is reflected as in the closed terrain case. The main advantage of this model is its more realism and the model has shown its capability to successfully capture real world traces through an appropriate choice of parameters [29]. 
International Journal of Wireless \& Mobile Networks (IJWMN) Vol. 6, No. 4, August 2014

\subsubsection{Working Day Mobility (WDM) Model}

In the WDM model, more real mobility patterns is achieved by modelling three major activities typically performed by humans during a working week: 1) sleeping at home, 2) working at the office, and 3) going out with friends in the evening. Different sub-models are derived corresponding to these three activities. The simulated nodes transition among these sub-models depends on the node type and the time of the day. Besides, the WDM model includes three different transport models. The nodes can move alone or in groups by walking, driving or traveling bus. This model offer nodes to move alone or in groups at different speeds and hence increases the heterogeneity of mobility pattern which significantly affect the performance of, e.g., routing protocols evaluation. Moreover, the concept of communities and social relationships included in the WDM model are not usually captured by simpler models such as RWP. This model provides inter-contact time and contact time distributions which follow closely the ones found in the traces from real-world measurements [22].

\subsubsection{General Social Mobility Model (GeSoMo)}

GeSoMo is a social-based mobility model which separates the core mobility model from the structural explanation of the social network underlying the simulation. This simple and elegant design principle gives GeSoMo generalizing power. GeSoMo accepts a social network as input data. Based on this, GeSoMo creates a mobility trace which is a schedule for the movement of each individual node. This trace generates meetings between the nodes according to their social relations. In this model, the attraction between nodes is defined based on node attraction, location attraction and node repulsion (i.e. negative attraction) [27]. In this model, any prevailing and future social network models can be used without changing GeSoMo itself. GeSoMo mobility model provides results that are coherent with a broad range of empirical data which describe real-world human social behaviour and mobility [9].

\subsection{Composite Mobility Models}

\subsubsection{Self-similar Least-Action Walk (SLAW)}

SLAW is one of the composite models that produce synthetic mobility traces which comprise all the five statistical features in various mobility settings. This also includes user-created virtual features, but no empirical information is available for these features. Virtual environments based on synthetic traces play important role for the performance evaluation of mobile networks as the networks are required to test in many diverse network settings when one design new network architecture. Performance evaluations of mobile routing protocols based on synthetic traces produced by SLAW demonstrate that SLAW gets the unique performance features of various routing protocols. Lee et al. [30] has developed SLAW depending on Global Positioning Systems traces of human walks that counted 226 daily traces collected from 101 volunteers in five different outdoor sites. Especially, many of these traces are derived considering people who have common interests such as students in the same university campuses and tourists in a theme park. By faithfully representing the properties present in these traces, SLAW can represent social contexts among walkers manifested by visits to common gathering places and walk patterns therein [31]. 
International Journal of Wireless \& Mobile Networks (IJWMN) Vol. 6, No. 4, August 2014

\subsubsection{Post-Disaster Mobility (PDM) model}

The PDM mimics the situation after a natural disaster. In fact, PDM includes mobility patterns depending on roles that node plays for a given city map. It models two main groups after a disaster: survivors, and rescue workers that aid survivors. PDM describes movement models for both groups. A post-disaster scenario is heavily dependent on the type of disaster occurred. For example, nodes will have different movement patterns in Hurricanes, tornadoes, earthquakes, and fire incident. PDM implemented on top of the ONE simulator, has four major movement types: inter-centre movement (repeated back and forth movement of supply vehicles between relief centres and main coordination centres), rescue worker movement (localized mobility of volunteers in distressed neighbourhoods), police patrols (cyclic police patrol movement among neighbourhoods), and emergency movement (vehicles attending to an emergency event). There are also several types of fixed nodes including centres, police stations, and hospitals, which act as meeting places of moving nodes that help relay packets among them. All moving entities and centres are equipped with ratio devices and these devices run DTN routing protocols [1].

\subsubsection{Localized Random Walk (LRAW) Mobility Model}

In the LRAW model, nodes are with a home cell which they tend to remain close to. The home cell includes a variety of places and structure ranging from home or den of a human or animal to the home base of a military unit. In this model, each node is assigned a fixed home cell. At each step each node makes a list of its neighbouring cells and chooses one with multinomial probability depending on each cell's distance from the node's home cell [32]. It is found that a node following the LRAW mobility model will have a double exponential (or Laplace) stationary distribution about the home cell. LRAW geographically biases node movement that can be modelled mathematically [33].

\subsubsection{Contact Graph Based (CG) Mobility Model}

In the contact graph model, a conceptual contact graph is formed mapping the sequence of actual contacts over time. The contacts are represented in a compact and tractable way as a weighted contact graph, where the weights (i.e., tie strengths) express how frequently and how long a pair of nodes is in contact. Given such a contact graph, one can use tools and metrics from social network analysis and graph theory (e.g., connectivity metrics, community detection, etc.) to quantify the amount of structure in the underlying mobility scenario. An important advantages is that this social structure of mobility is found as vital factors for the design of efficient DTN protocols [34, 35]. Hossmann et. Al [34] has constructed a conceptual contact graph from a campus mobility trace.

\section{COMParison}

\subsection{Comparison of Model Characteristics}

We provide comparison of model characteristics in Table 1(see next page) which shows several parameters. These parameters are the basic considerations for realistic mobility models. Each column corresponds to a parameter. The first column enlists each of the described mobility models. The main issues in selecting mobility models are whether or not a model is capable of determining the node's movement with a relation in time (time correlation) and a relation in space (spatial correlation). Then, the parameter node pattern relates to the type of mobility model for a node in its individual space. The parameter entity/group shows whether the model is applicable on single entity or whole system. Pause time is also another aspect disregarded in most mobility 
International Journal of Wireless \& Mobile Networks (IJWMN) Vol. 6, No. 4, August 2014

models but which is crucial to consider. Direction and speed are the regular aspects of mobility, while preferred locations are occasional.

Table 1: Comparison of the main properties of models

\begin{tabular}{|c|c|c|c|c|c|c|c|}
\hline Models & Node Pattern & Entity Group & Speed & Directica & \begin{tabular}{|c|} 
Pause \\
Time
\end{tabular} & \begin{tabular}{|c|} 
Time \\
Correlation
\end{tabular} & \begin{tabular}{|c|} 
Spatial \\
Correlation
\end{tabular} \\
\hline \multicolumn{8}{|c|}{ Random Mobility Models } \\
\hline $\mathrm{RW}$ & $\begin{array}{c}\text { Random } \\
\text { Unrealistic }\end{array}$ & Single & Random & Random & no & no & no \\
\hline RWP & $\begin{array}{c}\text { Random } \\
\text { Not similar with } \\
\text { Himnan }\end{array}$ & Single & $\begin{array}{c}\text { Uniformly } \\
\text { chosen }\end{array}$ & $\begin{array}{l}\text { Uniformly } \\
\text { chosen }\end{array}$ & yes & no & no \\
\hline $\mathrm{RD}$ & Random & single & Randam & $\begin{array}{l}\text { Uniformly chosen } \\
\text { In the range }[0, \Pi]\end{array}$ & yes & yes & yes \\
\hline RI & Real world model & Single & Uniform & $\begin{array}{l}\text { Uniformly } \\
\text { chosen }\end{array}$ & yes & & yes \\
\hline \multicolumn{8}{|c|}{ Map-Constrained Mobility Models } \\
\hline MBM & $\begin{array}{c}\text { Random within a } \\
\text { define area }\end{array}$ & $\begin{array}{l}\text { Conumumity } \\
\text { based }\end{array}$ & Uniform & $\begin{array}{c}\text { Uniformly } \\
\text { chosen }\end{array}$ & yes & yes & yes \\
\hline \begin{tabular}{|c|} 
SPBM \\
M
\end{tabular} & $\begin{array}{c}\text { Random within a } \\
\text { define area }\end{array}$ & $\begin{array}{c}\text { Commumity } \\
\text { based }\end{array}$ & Uniform & $\begin{array}{l}\text { Uniformly } \\
\text { chosen }\end{array}$ & yes & yes & yes \\
\hline RBMM & $\begin{array}{c}\text { Random within a } \\
\text { define area }\end{array}$ & $\begin{array}{l}\text { Commumity } \\
\text { based }\end{array}$ & Uniform & $\begin{array}{l}\text { Unifonnly } \\
\text { chosen }\end{array}$ & yes & yes & yes \\
\hline MDM & $\begin{array}{c}\text { Random within a } \\
\text { define area }\end{array}$ & $\begin{array}{l}\text { Comunumity } \\
\text { based }\end{array}$ & Uniform & $\begin{array}{l}\text { Uniformly } \\
\text { chosen }\end{array}$ & yes & yes & yes \\
\hline \multicolumn{8}{|c|}{ Social Mobility Models } \\
\hline CMM & $\begin{array}{l}\text { Based on social } \\
\text { attractiveness } \\
\text { towards } \\
\text { other nodes, but } \\
\text { includes } \\
\text { community } \\
\text { weight co such } \\
\text { decision. }\end{array}$ & $\begin{array}{l}\text { Community- } \\
\text { based, prochuct of } \\
\text { the decision of } \\
\text { individual nodes. }\end{array}$ & $\begin{array}{l}\text { Uniformly } \\
\text { chosen }\end{array}$ & $\begin{array}{l}\text { Intrinsic, } \\
\text { derived from the } \\
\text { target attraction } \\
\text { computation }\end{array}$ & yes & yes & yes \\
\hline CVTM & $\begin{array}{l}\text { Based on skewed } \\
\text { location } \\
\text { preferences, } \\
\text { And periodical } \\
\text { reappearance. }\end{array}$ & $\begin{array}{l}\text { Conmmunity- } \\
\text { based, } \\
\text { proctuct of the } \\
\text { decision of } \\
\text { indivichal nodes }\end{array}$ & $\begin{array}{l}\text { Uniformly } \\
\text { chosen }\end{array}$ & $\begin{array}{l}\text { Uniformly } \\
\text { chosen }\end{array}$ & yes & yes & yes \\
\hline WDM & $\begin{array}{c}\text { Based on all days } \\
\text { activities }\end{array}$ & $\begin{array}{l}\text { Commumity } \\
\text { based }\end{array}$ & $\begin{array}{c}\text { Uniformly } \\
\text { chosen }\end{array}$ & $\begin{array}{l}\text { Uniformily } \\
\text { chosen }\end{array}$ & yes & yes & yes \\
\hline GeSoMo & All social context & $\begin{array}{c}\text { Coummumity } \\
\text { based }\end{array}$ & $\begin{array}{c}\text { Uniformly } \\
\text { chosen }\end{array}$ & $\begin{array}{l}\text { Uniformly } \\
\text { chosen }\end{array}$ & yes & yes & yes \\
\hline \multicolumn{8}{|c|}{ Composite Mobility Models } \\
\hline SLAW & $\begin{array}{l}\text { Indivichal walker } \\
\text { model, based on } \\
\text { fractal waypoint } \\
\text { generation, } \\
\text { confined to } \\
\text { specific } \\
\text { areas }\end{array}$ & $\begin{array}{l}\text { Community- } \\
\text { based, } \\
\text { proctuct of the } \\
\text { decision of } \\
\text { individual nodes }\end{array}$ & $\begin{array}{c}\text { Intrinsic, } \\
\text { derived from } \\
\text { the } \\
\text { target } \\
\text { attraction } \\
\text { computatica }\end{array}$ & $\begin{array}{l}\text { Uniformly } \\
\text { chosen }\end{array}$ & yes & yes & yes \\
\hline PDM & $\begin{array}{l}\text { Specific part of } \\
\text { city }\end{array}$ & $\begin{array}{l}\text { Conumamity } \\
\text { based }\end{array}$ & $\begin{array}{c}\text { Uniformly } \\
\text { chosen }\end{array}$ & $\begin{array}{l}\text { Uniformly } \\
\text { chosen }\end{array}$ & yes & yes & yes \\
\hline LRAW & Local based & $\begin{array}{l}\text { Commamity } \\
\text { based }\end{array}$ & $\begin{array}{c}\text { Uniformly } \\
\text { chosen }\end{array}$ & $\begin{array}{l}\text { Uniformly } \\
\text { chosen }\end{array}$ & yes & yes & yes \\
\hline$C G$ & Correlated & $\begin{array}{l}\text { Commmumity } \\
\text { based }\end{array}$ & $\begin{array}{c}\text { Uniformly } \\
\text { chosen }\end{array}$ & $\begin{array}{l}\text { Uniformly } \\
\text { chosen }\end{array}$ & yes & yes & yes \\
\hline
\end{tabular}

\subsection{Comparison of Model Performance}

The Random Walk Mobility Model is basically suitable for a small input parameter (distance or time) and produces Brownian motion. The Random Walkway Point Mobility Model (RWP) is widely used in simulation studies of DTN protocols. RWP is found very flexible and offer opportunities to create realistic mobility patterns for the way people might move in such as a conference or museum sites. The main limitation is that node follows straight lines when travelling to the next chosen destination. Besides, this model often results in a non-uniform stationary node distribution. The factors that distinguish RWP from normal random walk model is that mobile nodes are more likely to cluster in the centre area of the simulation in Waypoint 
Mobility Model. The random direction distribution overcomes the problem of non-uniform distribution found in RWP. However, it is unexpected to be found that people would spread themselves evenly throughout an area (a building or a city) as well as people will not only pause at the edge of a given area. This problem is resolved by modifying Random Direction Mobility Model which allows mobile nodes (MNs) to pause for a while and change directions before touching the simulation boundary. However, this version is almost similar to the Random Walk Mobility Model with pause times. On the other hand, the Levy Walks is also very similar to random walk with an exception that the movement lengths and pause times are drawn from a power law distribution. They have been capable to provide inter-contact time distributions which are quite similar with many real world traces.

The Map-Based Movement model is derived from simple Random Walk model where nodes move randomly to specified directions on the map following the roads. In MBM, nodes move in the defined path. So they do not produce exact pattern like human. Random pauses occur at the waypoints. The Shortest Path Map-Based Movement model is an improved version of the Random Waypoint model where nodes choose a random destination decision points. Nodes move to that destination following the map-based shortest path. The route-based map mobility model is another up gradation of MBM. In this model, nodes travel randomly following the specified paths which is defined by the map of simulation scenario. This model is effectively used for simulation of movement of nodes in places such as buses and trams. On the other hand, Manhattan mobility model drive nodes over a grid like structure of horizontal and vertical lines.

CMM introduces several social movement aspects that bring this model closer to reality. Each node's movement is based on a function that captures social attraction to a target node. A group's movement is therefore simply a product of the decision of individual nodes. HCMM builds on top of CMM but in terms of node movement it adds the possibility of considering as target not only a node, but also a specific location. Hence, group movement is, similarly to CMM, a product of the decision of individual nodes. The main difference to CMM is therefore the movement function that can target nodes as well as locations. In addition to computing an attraction force towards nodes and/or places TVC captures also some human behaviour properties derived from traces, namely, the percentage of time nodes spends at specific locations, and preferred locations on different times and with different time granularity. This model incorporates the notion of aggregate pause time, by being able to capture the percentage of time nodes remain still in specific locations. WDM introduces communities and social relationships. This makes the strong relation between social models and random models. Existing social models is not flexible as each of them is implicitly restricted to a specific social network model. However, GeSoMo extracts the core mobility model from the structural explanation of the social network underlying the simulation. GeSoMo have generalizing power due to its simple and elegant design principle. Therefore, it has made to use existing and future social network models without changing GeSoMo itself.

SLAW has the particularity to rely on, as basis for an individual node's movement, a model that is based on fractal waypoint generation and which considers node's mobility within confined areas. SLAW captures movement through time and adds a strong relation towards a node's routine and the authors show that the resulting group mobility modelling follows a Power-Law distribution SLAW in addition considering the notion of pause time, but in an artificial way, by providing an average pause time to ensure that a whole trip (based on the individual walker model) completes in 12 hours. An interesting feature of SLAW is that while nodes are on the move towards a target, they may change their trajectory by picking, at random, new preferred locations (waypoints). PDM produces different role-based movements applying information of given city map. Travellers are always more likely to limit themselves to nearby destinations. This idea is addressed by the LRAW mobility model as well. An LRAW offer a designer to model a humans or animals cluster that live in the same area, or a platoon of soldiers operating in the neighbourhood of some home base. On the other hand, Contact Graph-Based Mobility Model 
represents a model that preserves the main aspects of real scenarios without over-specialization. This model is strictly focused on the graph with areas of different densities and therefore preserves the heterogeneous structure of real settings.

\section{FUTURE RESEARCH DIRECTIONS}

In our investigation, human is found as basic components of many DTN applications. Therefore, we should put more concentration to combine different human behaviour along with other modelling issues. As contact times are a microscopic property of human mobility, analysis built around them has tremendous significance in realistic mobility modelling especially when one departs from the exponential assumption, or a small amount of heterogeneity is introduced. In mobility modelling, human mobility and resulting contacts are assumed to be happen by intention and location. Secondly, social ties among nodes (e.g. friendship) guide a node to choose the destination and the timing of a mobility trip while location provides guidelines which path to be followed. This creates a rather intricate contact structure that is not readily observable at intercontact level. Therefore, a more abstract and macroscopic view of mobility is required to capture the set of node interrelations, in a more tractable manner. Therefore, in future researchers should put more concentration to combine different human behaviour along with aforementioned modelling issues. Furthermore, future investigations should also study other unexplored features of users such as their mobility patterns based on weekly schedule can be predicted to improve the performance of the forwarding algorithms. Further research is also required to identify the level of abstraction appropriate to produce good models. In this case goodness can be defined to mean how well generated traces statistically match collected mobility traces and how well models foresee the behaviour of different information distributing algorithms. In future, a more intelligent mobility model can be developed where each node have intelligence to select their own speed, direction and pause time considering the environment. At the top level of simulator, a framework can be developed to use different combination of mobility models with different level in one simulation. However, in this study, we have only provided a thorough literature review to extract the overview of DTN mobility models. In future, study based on simulation in a common simulator will reveal more acceptable comparison.

\section{CONCLUSION}

Recently mobility modelling has got enormous attention. Researchers are investigating different underlying mobility patterns. In our study, we analysed 16 popular mobility models to represent ins and outs regarding them. Random mobility is identified as simple to use, but usable in the small scale. They have also used as the base for other mobility models. Map Based model showed high performance to reflect practical traces. The models that incorporated human mobility characteristics, human mobility models, and human mobility prediction methods showed good result in simulation. However, more concentration to combine different human behaviour issues is required. Finally, we concluded that there are still a potential research issues to develop realistic DTN mobility models.

\section{REFERENCES}

[1] Uddin, Md Yusuf S., David M. Nicol, Tarek F. Abdelzaher, and Robin H. Kravets. "A post-disaster mobility model for delay tolerant networking." In Winter Simulation Conference, pp. 2785-2796. Winter Simulation Conference, 2009.

[2] Walker, Brenton D., T. Charles Clancy, and Joel K. Glenn. "Using localized random walks to model delay-tolerant networks." In Military Communications Conference, 2008. MILCOM 2008. IEEE, pp. 1-7. IEEE, 2008. 
International Journal of Wireless \& Mobile Networks (IJWMN) Vol. 6, No. 4, August 2014

[3] Hossmann, Theus, Thrasyvoulos Spyropoulos, and Franck Legendre. "Putting contacts into context: Mobility modeling beyond inter-contact times." In Proceedings of the Twelfth ACM International Symposium on Mobile Ad Hoc Networking and Computing, p. 18. ACM, 2011.

[4] Keränen, Ari, and Jörg Ott. "Increasing reality for dtn protocol simulations." Helsinki University of Technology, Tech. Rep (2007).

[5] Chaintreau, Augustin, Pan Hui, Jon Crowcroft, Christophe Diot, Richard Gass, and James Scott. "Impact of human mobility on opportunistic forwarding algorithms." Mobile Computing, IEEE Transactions on 6, no. 6 (2007): 606-620.

[6] Liang, Ben, and Zygmunt J. Haas. "Predictive distance-based mobility management for PCS networks." In INFOCOM'99. Eighteenth Annual Joint Conference of the IEEE Computer and Communications Societies. Proceedings. IEEE, vol. 3, pp. 1377-1384. IEEE, 1999.

[7] Hong, Xiaoyan, Mario Gerla, Guangyu Pei, and Ching-Chuan Chiang. "A group mobility model for ad hoc wireless networks." In Proceedings of the 2nd ACM international workshop on Modeling, analysis and simulation of wireless and mobile systems, pp. 53-60. ACM, 1999.

[8] Johnson, David B., and David A. Maltz. "Dynamic source routing in ad hoc wireless networks." In Mobile computing, pp. 153-181. Springer US, 1996.

AT\&T Research. Graphviz - Graph Visualization Software. http://www.graphviz.org/, 2008. [Online; accessed 19-May-2008].

[9] Petz, Agoston, Justin Enderle, and Christine Julien. "A framework for evaluating dtn mobility models." In Proceedings of the 2nd International Conference on Simulation Tools and Techniques, p. 94. ICST (Institute for Computer Sciences, Social-Informatics and Telecommunications Engineering), 2009.

[10] Miao, Jingwei. "Message dissemination in mobile delay tolerant networks." PhD diss., INSA de Lyon, 2013.

[11] C. Bettstetter, "Mobility modeling in wireless networks: categorization, smooth movement and border effects", ACM Mobile Computing and Commu. Rev., Jul. 2001.

[12] Bettstetter, Christian. "Mobility modeling in wireless networks: categorization, smooth movement, and border effects." ACM SIGMOBILE Mobile Computing and Communications Review 5, no. 3 (2001): 55-66.

[13] Royer, Elizabeth M., P. Michael Melliar-Smith, and Louise E. Moser. "An analysis of the optimum node density for ad hoc mobile networks." In Communications, 2001. ICC 2001. IEEE International Conference on, vol. 3, pp. 857-861. IEEE, 2001.

[14] Haas, Zygmunt J. "A new routing protocol for the reconfigurable wireless networks." In Universal Personal Communications Record, 1997. Conference Record., 1997 IEEE 6th International Conference on, vol. 2, pp. 562-566. IEEE, 1997.

[15] Zaninetti, Lorenzo, and Mario Ferraro. "On the truncated Pareto distribution with applications." Central European Journal of Physics 6, no. 1 (2008): 1-6.

[16] Karamshuk, Dmytro, Chiara Boldrini, Marco Conti, and Andrea Passarella. "Human mobility models for opportunistic networks." Communications Magazine, IEEE 49, no. 12 (2011): 157-165.

[17] Rhee, Injong, Minsu Shin, Seongik Hong, Kyunghan Lee, Seong Joon Kim, and Song Chong. "On the levy-walk nature of human mobility." IEEE/ACM Transactions on Networking (TON) 19, no. 3 (2011): 630-643.

[18] Keränen, Ari, Teemu Kärkkäinen, and Jörg Ott. "Simulating Mobility and DTNs with the ONE." Journal of Communications 5, no. 2 (2010): 92-105.

[19] Chen, Jing-Chao. "Dijkstra's shortest path algorithm." Journal of Formalized Mathematics 15 (2003).

[20] Anthru, Shermina V., and T. P. Jayakumar. "Opportunistic Routing Protocols In Human Working Day Model Delay Tolerant Networks." International Journal Of Engineering And Computer Science, Volume 3 Issue 5, 2014, PP 5960-5965. 
International Journal of Wireless \& Mobile Networks (IJWMN) Vol. 6, No. 4, August 2014

[21] Thakur, Gautam S., Udayan Kumar, Ahmed Helmy, and Wei-Jen Hsu. "Analysis of Spatio-Temporal Preferences and Encounter Statistics for DTN Performance." arXiv preprint arXiv:1007.0960 (2010).

[22] Bai, Fan, Narayanan Sadagopan, and Ahmed Helmy. "IMPORTANT: A framework to systematically analyze the Impact of Mobility on Performance of RouTing protocols for Adhoc NeTworks." In INFOCOM 2003. Twenty-Second Annual Joint Conference of the IEEE Computer and Communications. IEEE Societies, vol. 2, pp. 825-835. IEEE, 2003.

[23] Musolesi, Mirco, and Cecilia Mascolo. "Designing mobility models based on social network theory." ACM SIGMOBILE Mobile Computing and Communications Review 11, no. 3 (2007): 59-70.

[24] Boldrini, Chiara, and Andrea Passarella. "Hcmm: Modelling spatial and temporal properties of human mobility driven by users' social relationships." Computer Communications 33, no. 9 (2010): 10561074.

[25] Pirozmand, Poria, Guowei Wu, Behrouz Jedari, and Feng Xia. "Human mobility in opportunistic networks: Characteristics, models and prediction methods." Journal of Network and Computer Applications 42 (2014): 45-58.

[26] Hsu, Wei-jen, Thrasyvoulos Spyropoulos, Konstantinos Psounis, and Ahmed Helmy. "Modeling timevariant user mobility in wireless mobile networks." In INFOCOM 2007. 26th IEEE International Conference on Computer Communications. IEEE, pp. 758-766. IEEE, 2007.

[27] Mahendran, Veeramani, Sivaraman K. Anirudh, and C. Siva Ram Murthy. "A realistic framework for delay-tolerant network routing in open terrains with continuous churn." In Distributed Computing and Networking, pp. 407-417. Springer Berlin Heidelberg, 2011.

[28] Lee, Kyunghan, Seongik Hong, Seong Joon Kim, Injong Rhee, and Song Chong. "SLAW: self-similar least-action human walk." IEEE/ACM Transactions on Networking (TON) 20, no. 2 (2012): 515-529.

[29] Rhee, I., K. Lee, S. Hong, S. J. Kim, and S. Chong. "Demystifying the levy-walk nature of human walks." Techical Report, NCSU, http://netsrv. csc. ncsu. edu/export/Demystifying Levy Walk Patterns. pdf (2008).

[30] Walker, Brenton D., T. Charles Clancy, and Joel K. Glenn. "Using localized random walks to model delay-tolerant networks." In Military Communications Conference, 2008. MILCOM 2008. IEEE, pp. 1-7. IEEE, 2008.

[31] Walker, Brenton D., Joel K. Glenn, and T. Charles Clancy. "Analysis of simple counting protocols for delay-tolerant networks." In Proceedings of the second ACM workshop on Challenged networks, pp. 19-26. ACM, 2007.

[32] Gao, Wei, Qinghua Li, Bo Zhao, and Guohong Cao. "Multicasting in delay tolerant networks: a social network perspective." In Proceedings of the tenth ACM international symposium on Mobile ad hoc networking and computing, pp. 299-308. ACM, 2009.

[33] Hossmann, Theus, Thrasyvoulos Spyropoulos, and Franck Legendre. "Know thy neighbor: Towards optimal mapping of contacts to social graphs for dtn routing." In INFOCOM, 2010 Proceedings IEEE, pp. 1-9. IEEE, 2010.

[34] Shin, Minsu, Seongik Hong, and Injong Rhee. "DTN routing strategies using optimal search patterns." In Proceedings of the third ACM workshop on Challenged networks, pp. 27-32. ACM, 2008.

\section{Authors}

Md. Shahzamal has completed his B. Sc and M. Sc in 2006 and 2007 respectively in Applied Physics and Electronic Engineering, University of Rajshahi, Bangladesh. Currently he is senior scientific officer at the Institute of Electronics, Bangladesh Atomic Energy Commission. His research interests are Embedded System Design, Medical Instrumentation and Wireless Networking. He has published three international journal, several conference and internal review reports.

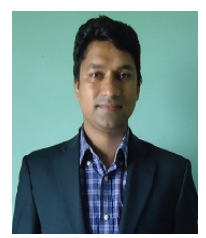


Md. Firoz Parvez has completed his B. Sc and M. Sc in 2011 and 2012 respectively in Applied Physics and Electronic Engineering, University of Rajshahi, Bangladesh. $\mathrm{He}$ is scientific officer at the Institute of Electronics, Bangladesh Atomic Energy Commission. His research interests are Embedded System and Wireless Networking.

Md. Anzan-Uz-Zaman has completed his B. Sc and M. Sc in 2007 and 2008 respectively in Applied Physics, Electronics \& Communication Engineering, Islamic University, Kustia, Bangladesh.At present he is senior scientific officer at the Institute of Electronics, Bangladesh Atomic Energy Commission. His research interests are VLSI, Thin Film, Embedded System Design and Wireless Networking. $\mathrm{He}$ has published several international journals, conferences and internal reports.
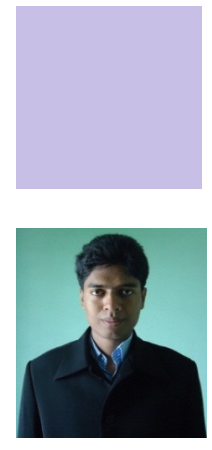

Dr. Md. Dulal Hossain is a Principal Engineer at the Institute of Computer Science, Ban gladesh Atomic Energy Commission. He received his PhD in Management Information Systems, MS in Electrical Engineering from KAIST, Korea and BS in Electrical \& Electronic Engineering from CUET, Bangladesh. Hossain's research interest includes Strategic use of IS/IT in organization, Knowledge/Innovation Management, National/Regional Innovation policy. His articles have appeared in journals such as Decision Support Systems, Electronic Commerce Research and

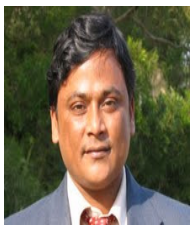
Applications, International Journal of Electronic Commerce, Asia Pacific Journal of Information Systems, Information Development, and Scientometrics. 